\title{
Cooperative behavior between oscillatory and excitable units: the peculiar role of positive coupling-frequency correlations
}

\author{
Bernard Sonnenschein ${ }^{1,2, a}$, Thomas K. DM. Peron ${ }^{3}$, Francisco A. Rodrigues ${ }^{4}$, \\ Jürgen Kurths ${ }^{1,2}$, and Lutz Schimansky-Geier ${ }^{1,2}$ \\ 1 Department of Physics, Humboldt-Universität zu Berlin, Newtonstrasse 15, 12489 Berlin, Germany \\ 2 Bernstein Center for Computational Neuroscience Berlin, Philippstrasse 13, 10115 Berlin, Germany \\ 3 Instituto de Física de São Carlos, Universidade de São Paulo, Avenida Trabalhador São Carlense 400, Caixa Postal 369, \\ CEP 13560-970 São Carlos, São Paulo, Brazil \\ 4 Departamento de Matemática Aplicada e Estatística, Instituto de Ciências Matemáticas e de Computação, \\ Universidade de São Paulo, Caixa Postal 668, 13560-970 São Carlos, São Paulo, Brazil
}

Received 28 April 2014 / Received in final form 18 June 2014

Published online 11 August 2014

(c) The Author(s) 2014. This article is published with open access at Springerlink.com

\begin{abstract}
We study the collective dynamics of noise-driven excitable elements, so-called active rotators. Crucially here, the natural frequencies and the individual coupling strengths are drawn from some joint probability distribution. Combining a mean-field treatment with a Gaussian approximation allows us to find examples where the infinite-dimensional system is reduced to a few ordinary differential equations. Our focus lies in the cooperative behavior in a population consisting of two parts, where one is composed of excitable elements, while the other one contains only self-oscillatory units. Surprisingly, excitable behavior in the whole system sets in only if the excitable elements have a smaller coupling strength than the selfoscillating units. In this way positive local correlations between natural frequencies and couplings shape the global behavior of mixed populations of excitable and oscillatory elements.
\end{abstract}

\section{Introduction}

Collective dynamics in biological systems is in general a complex behavior that results from the interplay of nonidentical, highly nonlinear and noisy elements [1]. Neuronal and cardiac rhythms for instance originate from interactions among pacemaker and excitable cells (see e.g. Refs. [2-5] and [6-9], respectively). Motivated by these facts, we investigate the collective dynamics of coupled non-identical elements, each being either excitable or selfoscillatory. The latter shall model the pacemaking cells in neuronal or cardiac tissues, for instance. Furthermore, in order to make the model more realistic, individual coupling strengths are allowed to be different. Our setting enables us to study how certain correlations between the dynamics and the couplings on the microscopic level affect the macroscopic behavior of the system. Many works addressed the latter kind of question recently, see e.g. references [10-26]. For interesting recent works that highlight the special interplay between dynamics and network structure in neuronal systems, we refer to $[27,28]$.

Of particular interest here are the works presented in references $[21,24]$. Zhang et al. considered Kuramoto oscillators coupled in a generalized complex network. Noteworthy, it was found that the crucial feature behind the

\footnotetext{
a e-mail: sonne@physik.hu-berlin.de
}

emergence of explosive synchronization ${ }^{1}$ is a positive correlation between the natural frequencies and the effective coupling strengths to the mean field [21]. Chen et al. studied effects of degree-frequency correlations in a population of FitzHugh-Nagumo neurons [24]. They extended in this way the finding of explosive synchronization to relaxation oscillators with two separated time scales.

The dynamical system that we study here puts emphasis on the phenomenon of excitability, both on the local and the global scale. Moreover, the coupling-frequency correlation considered in reference [21] shall motivate the specific formulation of our model. To this end, we investigate the noise-driven active rotator model introduced by Shinomoto and Kuramoto [29] with distributed natural frequencies and coupling strengths. Specifically, we analyze a system formed by two distinct parts of excitable and self-oscillating units, the first having subthreshold natural frequencies, while the other elements have frequencies above the excitation threshold.

Based on previous findings and numerical observations it is reasonable to approximate the phase distribution by a Gaussian with time-dependent mean and variance [30-33].

\footnotetext{
1 Explosive synchronization was coined by the finding of a discontinuous synchronization transition in scale-free networks of Kuramoto oscillators with bistability between incoherence and partial synchronization [11].
} 
Such an assumption has also been made, e.g., for coupled FitzHugh-Nagumo oscillators [34,35], integrate-andfire neurons [36], a general class of master equations [37] and delayed-coupled systems [38-40]. Within the Gaussian approximation the system's dimension can be reduced to four coupled first-order differential equations, which allows a thorough bifurcation analysis. On this basis, we distinguish the following global states: (i) a resting state, where the units are silent; (ii) a partially synchronized state, where a macroscopic fraction of the units fire in synchrony and (iii) an incoherent state, where all the units fire asynchronously. Finally, bistability between the resting and the partially synchronized or the incoherent state is reported.

We find that heterogeneity both in the natural frequencies and the coupling strengths impedes synchronization. However, a counterintuitive phenomenon is found on top of this. A positive coupling-frequency correlation where the self-oscillatory units possess a stronger coupling than the excitable elements, brings the whole system into an excitable state. Since individual coupling strengths constrain how strongly single elements can feel the mean field, the emergence of the excitable behavior on the global scale crucially depends on how strongly the self-oscillating units are influenced by the excitable elements. Bistable behavior is only found if the coupling-frequency correlation is sufficiently strong.

As an aside, our theory also yields analytical findings for the stochastic Kuramoto model where temporal fluctuations act on the frequencies and where the only source of quenched disorder is provided by different coupling strengths. We discover that the mean-field amplitude of the oscillators with weaker coupling can scale anomalously in dependence on the average coupling strength, giving rise to chimera-like states (see Appendix A).

The paper is organized as follows: in Section 2 we present the model and explain its basic properties. Section 3 is devoted to the mean-field treatment and to the derivation of the Fokker-Planck equation for the probability distribution of the phases. In Section 4 we prepare the low-dimensional behavior of the model through the Gaussian approximation technique, and in Section 5 we work out a specific example. Numerical results thereby corroborate the theoretical findings. Final conclusions are drawn in Section 6.

\section{Model}

Consider a population of noise-driven active rotators [29], where the dynamics of individual phases $\phi_{i}(t)$ follows

$$
\dot{\phi}_{i}=\omega_{i}-a \sin \phi_{i}+\frac{K_{i}}{N} \sum_{j=1}^{N} \sin \left(\phi_{j}-\phi_{i}\right)+\xi_{i}(t) .
$$

The units are indexed by $i=1, \ldots, N$. The parameter $a$, which determines the excitation threshold, is the same for all rotators. Natural frequencies are denoted by $\omega_{i}$.
Each element is coupled to the others with an individual strength, $K_{i}$. We will assume that the individual frequencies and coupling strengths are random numbers that are drawn from the same joint probability distribution $P(\omega, K)$, independently between the elements. In addition, we assume that the initial phases of the active rotators $\phi_{i}^{0}$ at the starting time $t^{0}$ are independent and given by a distribution density $P_{\text {in }}\left(\phi^{0}\right)$.

We emphasize that the values for the $\omega$ 's and $K$ 's are chosen initially and then stay fixed during the whole evolution of the system. They represent frozen random variables ("quenched disorder"), which shall be some real numbers. We do not consider repulsive interactions here, that is the coupling strengths are non-negative.

The terms $\xi_{i}(t)$ shall model the accumulated effect of various sources of temporal fluctuations that may result from a noisy environment, cell-intrinsic noise and stochasticity in the interactions. Lumped together, one may assume zero mean Gaussian white noise sources [41,42]. Then one has

$$
\left\langle\xi_{i}(t)\right\rangle=0, \quad\left\langle\xi_{i}(t) \xi_{j}\left(t^{\prime}\right)\right\rangle=2 D \delta_{i j} \delta\left(t-t^{\prime}\right),
$$

where the second relation expresses the lack of memory in the noise and that noise at one element is independent from the other ones. The angular brackets denote averages over different realizations of the noise and $D$ is the noise intensity.

For an isolated element without additive noise, $\dot{\phi}_{i}=$ $\omega_{i}-a \sin \left(\phi_{i}\right)$, the excitable behavior is easily understood. For $|a|>\omega_{i}$ the stable equilibrium is located at $\phi_{i}^{\infty}=\arcsin \left(\omega_{i} / a\right)$, and the unit needs a sufficiently strong perturbation in order to make a big excursion. Noise can play this role driving the system to escape from the resting state $\phi_{i}^{\infty}$. An escape event corresponds to the release of a single spike [29]. For $|a|<\omega_{i}$ the element shows oscillatory behavior with frequency $\sqrt{\omega_{i}^{2}-a^{2}}$. It has to be emphasized that the phase variable $\phi$ does not rotate uniformly; it is slowest near $\phi=\pi / 2$ and fastest near $\phi=3 \pi / 2$.

\section{Mean-field theory}

Instead of the Langevin equations (1) the system of $N$ coupled active rotators can be described by the joint probability density

$$
\mathcal{P}_{N}\left(\phi, t ; \phi^{0}, t^{0} ; \boldsymbol{\omega}, \boldsymbol{K}\right)
$$

The vector $\phi=\left(\phi_{1}, \ldots, \phi_{N}\right)$ is built from the phases of $N$ rotators at time $t$, and $\phi^{0}=\left(\phi_{1}^{0}, \ldots, \phi_{N}^{0}\right)$ consists of their values at the initial time $t^{0}$. The time-independent vectors $\boldsymbol{\omega}=\left(\omega_{1}, \ldots, \omega_{N}\right)$ and $\boldsymbol{K}=\left(K_{1}, \ldots, K_{N}\right)$ are composed of, respectively, the natural frequencies and the coupling strengths of the $N$ rotators. Normalization requires

$$
\int_{0}^{2 \pi} d^{N} \boldsymbol{\phi} \int_{0}^{2 \pi} d^{N} \boldsymbol{\phi}^{0} \int_{-\infty}^{+\infty} d^{N} \boldsymbol{\omega} \int_{0}^{+\infty} d^{N} \boldsymbol{K} \mathcal{P}_{N}=1
$$


For rotators with given $\boldsymbol{\omega}$ and $\boldsymbol{K}$, this joint probability distribution is related with the conditional probability density $p_{N}\left(\phi, t \mid \phi^{0}, t^{0} ; \boldsymbol{\omega}, \boldsymbol{K}\right)$ from the initial state $\phi^{0}$ at $t^{0}$ to the present phases $\phi$ at time $t$ as:

$$
\begin{aligned}
\mathcal{P}_{N}\left(\phi, t ; \phi^{0}, t^{0} ; \boldsymbol{\omega}, \boldsymbol{K}\right) \\
\quad=p_{N}\left(\phi, t \mid \phi^{0}, t^{0} ; \boldsymbol{\omega}, \boldsymbol{K}\right) P_{N}\left(\phi^{0}, t^{0} ; \boldsymbol{\omega}, \boldsymbol{K}\right) .
\end{aligned}
$$

By assuming independent initial phases and pairs $\omega_{i}$ and $K_{i}$ at the nodes, we can factorize

$$
P_{N}\left(\phi^{0}, t^{0} ; \boldsymbol{\omega}, \boldsymbol{K}\right)=\prod_{i=1}^{N} P_{\text {in }}\left(\phi_{i}^{0}\right) P\left(\omega_{i}, K_{i}\right) .
$$

The joint probability density is governed by a linear Fokker-Planck equation (FPE) which describes the evolution of the population from time $t^{0}$ to time $t>t^{0}$ [43]:

$$
\begin{aligned}
\frac{\partial \mathcal{P}_{N}}{\partial t}= & D \sum_{i=1}^{N} \frac{\partial^{2} \mathcal{P}_{N}}{\partial \phi_{i}^{2}}-\sum_{i=1}^{N} \frac{\partial}{\partial \phi_{i}} \mathcal{P}_{N} \\
& \times\left[\omega_{i}-a \sin \phi_{i}+\frac{K_{i}}{N} \sum_{j=1}^{N} \sin \left(\phi_{j}-\phi_{i}\right)\right]
\end{aligned}
$$

The usual way to proceed is to introduce reduced probability densities $\mathcal{P}_{n}$ with index $n=1,2, \ldots, N-1$ by integrating $\mathcal{P}_{N}$ over a subset of variables and parameters. Since all rotators are identical in their dynamic behavior with respect to the specific frequencies and coupling constants, we take exemplarily rotators with labels $i=1, \ldots, n$ and integrate over respective variables and parameters with numbers $i>n$. This defines the reduced probability densities with integration boundaries as in (4):

$$
\begin{aligned}
\mathcal{P}_{n}\left(\phi_{1}, t ; \phi_{1}^{0}, t^{0} ; \omega_{1}, K_{1} ; \ldots ; \phi_{n}, t ; \phi_{n}^{0}, t^{0} ; \omega_{n}, K_{n}\right) \\
\quad=\int \prod_{i=n+1}^{N}\left(d \phi_{i} d \phi_{i}^{0} d \omega_{i} d K_{i}\right) \mathcal{P}_{N}\left(\phi, t ; \phi^{0}, t^{0} ; \boldsymbol{\omega}, \boldsymbol{K}\right) .
\end{aligned}
$$

To obtain the dynamics for these densities, we integrate the FPE (7) over the corresponding subset of the variables and the other quantities. Then one is left with a set of coupled differential equations, akin to a Bogoliubov-BornGreen-Kirkwood-Yvon (BBGKY) hierarchy. Truncating this hierarchy at some $n$ leads to a reduced description.

Specifically here, we will be interested in the one-oscillator probability density $\mathcal{P}_{1}$. Therefore, we integrate the FPE (7) over the $N-1$ phases $\phi_{2}, \ldots, \phi_{N}$, their initial values $\phi_{2}^{0}, \ldots, \phi_{N}^{0}$, the natural frequencies $\omega_{2}, \ldots, \omega_{N}$ and the coupling strengths $K_{2}, \ldots, K_{N}$. This yields

$$
\begin{aligned}
\frac{\partial \mathcal{P}_{1}}{\partial t}= & D \frac{\partial^{2} \mathcal{P}_{1}}{\partial \phi_{1}^{2}}-\frac{\partial}{\partial \phi_{1}}\left(\omega_{1}-a \sin \phi_{1}\right) \mathcal{P}_{1}-\frac{K_{1}(N-1)}{N} \\
& \times \frac{\partial}{\partial \phi_{1}} \int d \phi_{2} \int d \phi_{2}^{0} \int d \omega_{2} \int d K_{2} \sin \left(\phi_{2}-\phi_{1}\right) \\
& \times \mathcal{P}_{2}\left(\phi_{1}, \phi_{2}, t ; \phi_{1}^{0}, \phi_{2}^{0}, t^{0} ; \omega_{1}, K_{1}, \omega_{2}, K_{2}\right)
\end{aligned}
$$

Note that $\mathcal{P}_{1}\left(\phi_{1}, t ; \phi_{1}^{0}, t^{0} ; \omega_{1}, K_{1}\right)$ relates hierarchically to $\mathcal{P}_{2}\left(\phi_{1}, \phi_{2}, t ; \phi_{1}^{0}, \phi_{2}^{0}, t^{0} ; \omega_{1}, K_{1}, \omega_{2}, K_{2}\right)$ being the two-oscillator distribution.

The investigation of coupling-coupling or frequencyfrequency correlations shall remain a topic for future research. However, we will allow dependencies between the natural frequency and the coupling strength at each node, given by the joint distribution $P(\omega, K)$. Having this in mind, we will assume that the dynamical correlations between the phases of two arbitrarily chosen oscillators can be discarded as follows:

$$
\begin{aligned}
& \mathcal{P}_{2}\left(\phi_{1}, \phi_{2}, t ; \phi_{1}^{0}, \phi_{2}^{0}, t^{0} ; \omega_{1}, K_{1}, \omega_{2}, K_{2}\right) \\
& \quad \equiv \mathcal{P}_{1}\left(\phi_{1}, t ; \phi_{1}^{0}, t^{0} ; \omega_{1}, K_{1}\right) \mathcal{P}_{1}\left(\phi_{2}, t ; \phi_{2}^{0}, t^{0} ; \omega_{2}, K_{2}\right) .
\end{aligned}
$$

This corresponds essentially to the lowest-order truncation of the BBGKY hierarchy. In particular, equation (8) becomes closed but nonlinear in $\mathcal{P}_{1}$. Remarkably, in the thermodynamic limit of infinitely many elements, $N \rightarrow \infty$, such a truncation can be justified in a rigorous way for various systems (for recent overviews along with new results, cf. Refs. [44,45]). The argument goes back to Boltzmann's "Stosszahlansatz", which was later rigorously formalized by Kac with the concept of "propagation of molecular chaos" [46]. In the light of those achievements (see also $[47,48]$ and Refs. therein), (9) can be considered to be exact in the thermodynamic limit.

Henceforth we neglect the indices at $\phi, \omega$ and $K$, as the underlying assumption in the mean-field approach is that rotators with the same natural frequency and coupling strength are statistically identical. Moreover, we proceed with the conditional form of the one-oscillator probability density $p_{1}$, which is obtained from $p_{N}$ (see Eq. (5)) after appropriate integration [49]. For every given pair $(\omega, K)$, the expression $p_{1}\left(\phi, t \mid \phi^{0}, t^{0} ; \omega, K\right) d \phi$ denotes the fraction of oscillators, which start with the phase $\phi^{0}$ at time $t^{0}$ and then have a phase value between $\phi$ and $\phi+d \phi$ at time $t$. It follows from integration of the $p_{N}$ and from an average over frequency and coupling constants of the other units of the ensemble. Accordingly, the normalization $1=$ $\int_{0}^{2 \pi} d \phi p_{1}\left(\phi, t \mid \phi^{0}, t^{0} ; \omega, K\right) \forall \phi^{0}, \omega, K$ must be satisfied.

For the dynamical evolution of $p_{1}\left(\phi, t \mid \phi^{0}, t^{0} ; \omega, K\right)$ one gets the following nonlinear Fokker-Planck [50] or McKean-Vlasov equation:

$$
\frac{\partial p_{1}}{\partial t}=-\frac{\partial}{\partial \phi} v_{\omega, K}(\phi, t) p_{1}+D \frac{\partial^{2} p_{1}}{\partial \phi^{2}}
$$

Nonlinearity enters equation (10) through the mean increment of the phase per unit time, i.e.

$$
v_{\omega, K}(\phi, t) \equiv \omega-a \sin \phi+r K \sin (\Theta-\phi),
$$

which depends on the density $p_{1}$ via the mean-field amplitude $r(t)$ and phase $\Theta(t)$,

$$
r(t) e^{i \Theta(t)}=\left\langle\left\langle r_{\omega^{\prime}, K^{\prime}}(t) e^{i \Theta_{\omega^{\prime}, K^{\prime}}(t)}\right\rangle\right\rangle .
$$

The averages $\langle\langle\ldots\rangle\rangle \equiv \int d \omega^{\prime} \int d K^{\prime} \ldots P\left(\omega^{\prime}, K^{\prime}\right)$ connect in a superposed manner the global with the following local 
mean-field variables,

$$
\begin{aligned}
r_{\omega, K}(t) e^{i \Theta_{\omega, K}(t)}= & \int_{0}^{2 \pi} d \phi \int_{0}^{2 \pi} d \phi^{0} e^{i \phi} \\
& \times p_{1}\left(\phi, t \mid \phi^{0}, t^{0} ; \omega, K\right) P_{\text {in }}\left(\phi^{0}\right) .
\end{aligned}
$$

The set of equations (10)-(13) has to be solved with the initial condition for the transition probability density

$$
p_{1}\left(\phi, t^{0} \mid \phi^{0}, t^{0} ; \omega, K\right)=\delta\left(\phi-\phi^{0}\right), \forall \omega, K .
$$

Alternatively, we can formulate the problem in terms of a nonlinear FPE for the marginal density of the phase $\phi$ at time $t$,

$$
p_{1}(\phi, t \mid \omega, K)=\int_{0}^{2 \pi} d \phi^{0} p_{1}\left(\phi, t \mid \phi^{0}, t^{0} ; \omega, K\right) P_{\text {in }}\left(\phi^{0}\right) .
$$

Specifically, this marginal density replaces the conditional probability density in the nonlinear FPE (10) and in equation (13) via integration over the initial phases. Then we have an initial value problem that has to be solved, in agreement with former assumptions, with the initial condition

$$
p_{1}\left(\phi, t^{0} \mid \omega, K\right)=P_{\text {in }}\left(\phi^{0}\right), \forall \omega, K .
$$

In the derivation of (10)-(13), we did not drop the dependence of the conditional probability density on the initial state. Since the FPE is nonlinear, the temporal evolution of the mean field and of the drift term in (10) can sensitively depend on the initial distribution of the phases. We also note that the assumption of propagation of chaos appears to be problematic in sparsely connected networks, cf. Section 7 in reference [49] for numerical findings.

We remark that the nonlinear FPE (10) comprises a large system of coupled partial differential equations. The nodes with coinciding pairs of frequency and coupling constants can be interpreted as one species. Every species obeys the FPE with the corresponding $\omega$ and $K$. They contribute with subfields given by equation (13) in accordance with their emergence to the mean field (12). The latter is given by the probability density $P(\omega, K)$ about which the subfields are averaged.

\section{Fourier series expansion and Gaussian approximation}

We proceed to study the evolution of the marginal density $p_{1}(\phi, t \mid \omega, K)$, equation (15). Since it is $2 \pi$-periodic in $\phi$, we can first write a Fourier series expansion:

$$
p_{1}(\phi, t \mid \omega, K)=\frac{1}{2 \pi} \sum_{n=-\infty}^{+\infty} \rho_{n}(t \mid \omega, K) e^{-i n \phi},
$$

with $\rho_{0}=1$ and $\rho_{-n}=\rho_{n}^{*}$.

Inserting (17) into (10), multiplying by $\exp (i m \phi)$, $m \in \mathbb{Z}$ and collecting the non-zero terms after integration over $\phi$, one obtains an infinite chain of coupled complexvalued differential equations for the Fourier coefficients $\rho_{n}(t \mid \omega, K)$. That is, for every pair $(\omega, K)$ we can write

$$
\begin{aligned}
\frac{\dot{\rho}_{n}(t \mid \omega, K)}{n}= & \frac{a}{2}\left[\rho_{n-1}(t \mid \omega, K)-\rho_{n+1}(t \mid \omega, K)\right] \\
& -(D n-i \omega) \rho_{n}(t \mid \omega, K) \\
& +\frac{K}{2}\left[\rho_{n-1}(t \mid \omega, K)\left\langle\left\langle\rho_{1}\left(t \mid \omega^{\prime}, K^{\prime}\right)\right\rangle\right\rangle\right. \\
& \left.-\rho_{n+1}(t \mid \omega, K)\left\langle\left\langle\rho_{-1}\left(t \mid \omega^{\prime}, K^{\prime}\right)\right\rangle\right\rangle\right] .
\end{aligned}
$$

An additional average appears if one considers complex networks in a coarse-grained way [32]. While (18) provides an exact representation of the system, it is not possible to derive the solutions in an explicit way due to its hierarchical character. Since the Fourier coefficients rapidly decay with growing $n$, one can get accurate results by truncating the hierarchy at a large enough $n$. Here we aim for an approximate dimensionality reduction that allows bifurcation analysis or even explicit solutions in important limiting cases. This is the topic of the next sections.

We first seek a closure of the infinite set of equations (18). The Ott-Antonsen ansatz [51] achieves this in an exact manner for deterministic ensembles of coupled phase oscillators. Unfortunately, for the case with temporal fluctuations the direct application of the Ott-Antonsen ansatz is not possible, and we are unaware of its appropriate modifications.

Here we use instead a Gaussian approximation (GA): we assume that in every subset of oscillators with the same individual quantities $(\omega, K)$, the distribution of the phases at every moment of time is Gaussian with mean $m_{\omega, K}(t)$ and variance $\sigma_{\omega, K}^{2}(t)[31,32]$.

Consider separately the real and imaginary parts of the Fourier coefficients (17), that is

$$
\rho_{n}(t \mid \omega, K) \equiv c_{n}(t \mid \omega, K)+i s_{n}(t \mid \omega, K)
$$

In the thermodynamic limit $N \rightarrow \infty$ the GA then yields

$$
\begin{aligned}
& c_{n}(t \mid \omega, K)=\exp \left[-n^{2} \sigma_{\omega, K}^{2}(t) / 2\right] \cos \left[n m_{\omega, K}(t)\right], \\
& s_{n}(t \mid \omega, K)=\exp \left[-n^{2} \sigma_{\omega, K}^{2}(t) / 2\right] \sin \left[n m_{\omega, K}(t)\right] .
\end{aligned}
$$

As a result, all $c_{n}$ and $s_{n}$ are given as combinations of $c_{1}$ and $s_{1}: c_{2}=c_{1}^{4}-s_{1}^{4}, s_{2}=2 s_{1} c_{1}\left(s_{1}^{2}+c_{1}^{2}\right)$, etc. [31].

By transforming the variables $\left\{c_{1}(t \mid \omega, K), s_{1}(t \mid \omega, K)\right\}$ to the first two cumulants of the Gaussian distribution, $\left\{m_{\omega, K}(t), \sigma_{\omega, K}^{2}(t)\right\}$, we obtain the following pair of differential equations:

$$
\left\{\begin{aligned}
\dot{m}_{\omega, K}= & \omega-\exp \left(-\sigma_{\omega, K}^{2} / 2\right) \cosh \sigma_{\omega, K}^{2}\left[a \sin m_{\omega, K}\right. \\
& -K\left\langle\left\langle\exp \left(-\sigma_{\omega^{\prime}, K^{\prime}}^{2} / 2\right)\right.\right. \\
& \left.\left.\left.\times \sin \left(m_{\omega^{\prime}, K^{\prime}}-m_{\omega, K}\right)\right\rangle\right\rangle\right] \\
\dot{\sigma}_{\omega, K}^{2} / 2= & D-\exp \left(-\sigma_{\omega, K}^{2} / 2\right) \sinh \sigma_{\omega, K}^{2}\left[a \cos m_{\omega, K}\right. \\
& +K\left\langle\left\langle\exp \left(-\sigma_{\omega^{\prime}, K^{\prime}}^{2} / 2\right)\right.\right. \\
& \left.\left.\left.\times \cos \left(m_{\omega^{\prime}, K^{\prime}}-m_{\omega, K}\right)\right\rangle\right\rangle\right]
\end{aligned}\right.
$$


Thus, for a continuous coupling-frequency distribution $P(\omega, K)$ the reduced system is still infinite-dimensional, because for any pair $(\omega, K)$ one has to solve the two differential equations (21), and all of those are coupled through the averages $\langle\langle\ldots\rangle\rangle$. In order to obtain a low-dimensional system, we need to continue with a discrete couplingfrequency distribution $P(\omega, K)$ with a finite number of different $\omega$ 's and $K$ 's. Indeed, interesting example systems are readily found, as shown in the next section.

Before coming to the integral part of our analysis, we would like to mention that one can also perform a variable transformation to the local mean-field variables:

$$
\left\{\begin{aligned}
\dot{r}_{\omega, K}= & -r_{\omega, K} D+\frac{1-r_{\omega, K}^{4}}{2}\left[a \cos \Theta_{\omega, K}\right. \\
& \left.+K\left\langle\left\langle r_{\omega^{\prime}, K^{\prime}} \cos \left(\Theta_{\omega^{\prime}, K^{\prime}}-\Theta_{\omega, K}\right)\right\rangle\right\rangle\right], \\
\dot{\Theta}_{\omega, K}= & \omega-\frac{r_{\omega, K}^{-1}+r_{\omega, K}^{3}}{2}\left[a \sin \Theta_{\omega, K}\right. \\
& \left.-K\left\langle\left\langle r_{\omega^{\prime}, K^{\prime}} \sin \left(\Theta_{\omega^{\prime}, K^{\prime}}-\Theta_{\omega, K}\right)\right\rangle\right\rangle\right] .
\end{aligned}\right.
$$

Note that the mean phases are not defined in the case of vanishing mean-field amplitudes.

Let us briefly recapitulate. We have derived an approximate lower-dimensional description for the infinite-dimensional system (1), expressed by $\dot{c}_{1}(t \mid \omega, K), \dot{s}_{1}(t \mid \omega, K)$ or $\dot{m}_{\omega, K}(t), \quad \dot{\sigma}_{\omega, K}^{2}(t)$ or $\dot{r}_{\omega, K}(t)$, $\dot{\Theta}_{\omega, K}(t)$. All three descriptions are equivalent. Now one could directly plug in a correlation between the coupling strength $K$ and the natural frequency $\omega$ as proposed by Zhang et al. [21]. Many interesting examples are conceivable, and we expect further fruitful investigations building on what we have just derived.

In the past, understanding the effects of heterogeneity benefited immensely by dividing the whole system into two subpopulations (see e.g. Refs. [32,52-57]). This strategy will also be adopted in the next section.

\section{Mixed population of excitable and self-oscillatory elements}

On the basis of the reduced description derived in the previous section, we will now turn our attention to an interesting example that allows a detailed analysis. We consider a mixed population consisting of two equally sized constituents; one half is chosen to be excitable and the other half shall be self-oscillating. This is realized by choosing one natural frequency below and the other one above the excitation threshold. Furthermore, both subpopulations shall have their own coupling strengths. Hence, for the coupling-frequency distribution we take a sum of two delta functions, $P(\omega, K)=p \delta\left[(\omega, K)-\left(\omega_{1}, K_{1}\right)\right]+(1-$ $p) \delta\left[(\omega, K)-\left(\omega_{2}, K_{2}\right)\right]$, with $p=0.5,0<\omega_{1}<1, \omega_{2}>1$, and $K_{1,2}>0$. In particular, we proceed with the following four-dimensional system (cf. Eq. (21)):

$$
\left\{\begin{aligned}
\dot{m}_{1}= & \omega_{1}-e^{-\sigma_{1}^{2} / 2} \cosh \sigma_{1}^{2}\left[\sin m_{1}\right. \\
& \left.+\left(K_{1} / 2\right) e^{-\sigma_{2}^{2} / 2} \sin \left(m_{1}-m_{2}\right)\right] \\
\dot{\sigma}_{1}^{2} / 2= & D-e^{-\sigma_{1}^{2} / 2} \sinh \sigma_{1}^{2}\left\{\cos m_{1}\right. \\
& \left.+\left(K_{1} / 2\right)\left[e^{-\sigma_{1}^{2} / 2}+e^{-\sigma_{2}^{2} / 2} \cos \left(m_{1}-m_{2}\right)\right]\right\},
\end{aligned}\right.
$$

where $\omega_{1}=1-\Delta \omega / 2$ and $K_{1}=K_{0}-\Delta K / 2$. The equations for $\dot{m}_{2}$ and $\dot{\sigma}_{2}^{2}$ are similar; just interchange 1's with 2 's, and set $\omega_{2}=1+\Delta \omega / 2, K_{2}=K_{0}+\Delta K / 2$. Indices $i=1,2$ are abbreviations for $\left\{\omega_{i}, K_{i}\right\}$. Henceforth, we call the differences in the natural frequencies and coupling strengths frequency mismatch $(\Delta \omega)$ and coupling mismatch $(\Delta K)$, respectively. Note that the above choice is such that the average frequency and coupling strength are not affected by the mismatches. Equations for the four mean-field variables follow mutatis mutandis from equation (22).

Similar problems were addressed in the context of oscillatory systems, where parts are inactivated due to aging [58-60]. Another mixed population of excitable and "driver" units was studied by Alonso and Mindlin [61]. Finally, the recent work [4] puts forward a detailed analysis of coupled theta neurons where both inherently spiking and excitable neurons are present.

From now on we study the collective behavior in system (23) with the help of MATCONT [62], namely in dependence on four dimensionless parameters: the noise intensity $D$, the frequency mismatch $\Delta \omega$, the coupling mismatch $\Delta K$, and the average coupling strength $K_{0}$.

The frequency mismatch $\Delta \omega$ is varied in the interval $(0,2)$, restricting to positive natural frequencies. The coupling mismatch $\Delta K$ can take values between $\left(-2 K_{0}, 2 K_{0}\right)$. Positive (negative) values of $\Delta K$ can be referred to as positive (negative) coupling-frequency correlations, as long as there is a frequency mismatch $\Delta \omega>0$. Similar as in reference [63], we focus here first on $K_{0}=4$; in Appendix $\mathrm{C}$ we show results for smaller average coupling strengths.

Coupled excitable elements stay at rest, if they cannot globally surpass the excitation threshold. If they do, the question then is whether a macroscopic fraction of them fires in synchrony, which amounts to a partially synchronized state, or whether the firing is completely incoherent among the elements. Figure 1 depicts the Hopf and saddle-node bifurcations that delineate those three states. Additional Hopf and saddle-node bifurcations that come after unstable equilibria can be neglected, because they do not affect the dynamics. As one would expect, increased frequency and coupling mismatches impede the emergence of collectively synchronized oscillations. Specifically, above the Hopf bifurcation line the oscillatory units fire incoherently, while below the Hopf line a synchronized firing sets in. Interestingly, a positive coupling-frequency correlation gives rise to a qualitative change in the global dynamics, since the saddle-node bifurcation shows up for $\Delta K>0$. Indeed the critical value for this phenomenon is found to 

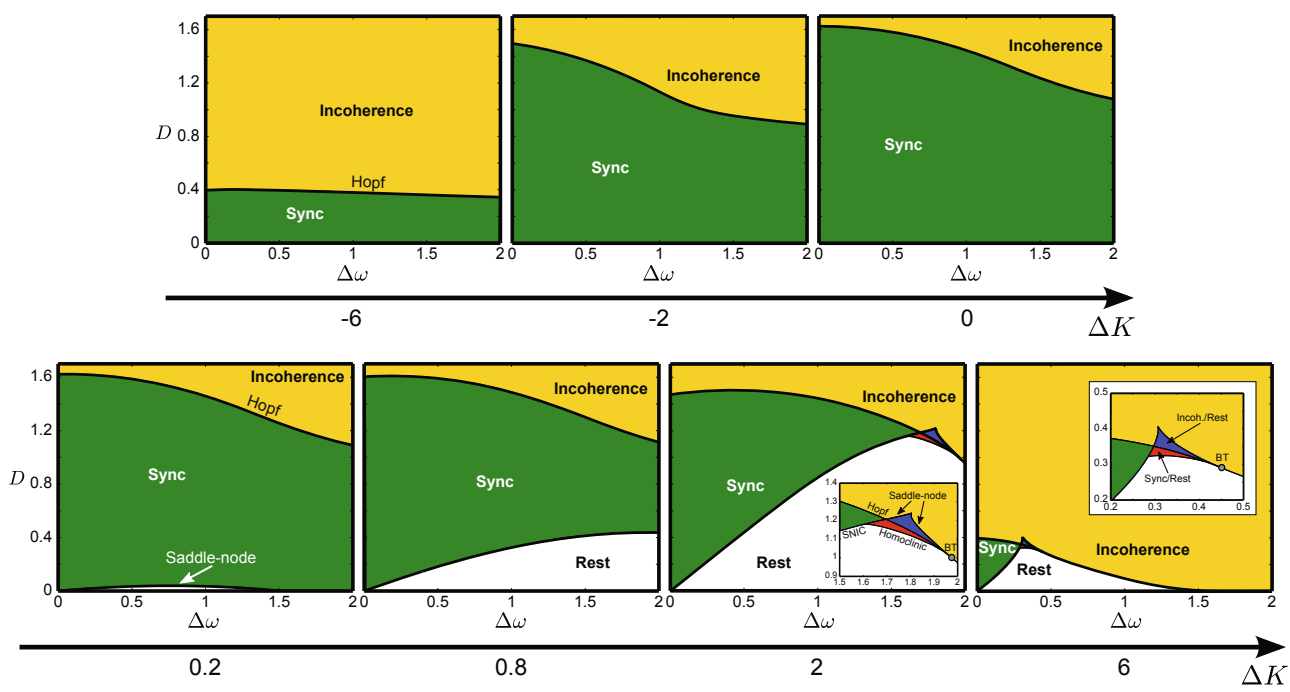

Fig. 1. Hopf and saddle-node bifurcations in the plane spanned by the noise intensity $D$ and the frequency mismatch $\Delta \omega$, as obtained from a bifurcation analysis of the reduced system (23). Changes in the bifurcation diagram are shown as a function of the coupling mismatch $\Delta K$, with a fixed average coupling strength of $K_{0}=4$. Green shaded areas with label "Sync" represent the partially synchronized state, while in the yellow shaded regions no synchronized oscillations are found. In the white areas the system is at rest, in which the excitable units do not fire. For $\Delta K=2$ and $\Delta K=6$ insets show in more detail the parameter regions that correspond to bistable dynamics. The latter are found between the saddle-node bifurcation curves and the homoclinic bifurcation line which emanates from a Bogdanov-Takens bifurcation (BT). Moreover, the bistable region is separated into two parts by the Hopf bifurcation line. In the red area below the Hopf line partially synchronized and resting state coexist, while in the blue area resting and incoherence coexist.

equal $\Delta K_{c}=0$. This is visualized in Figure 1 for a small coupling mismatch of $\Delta K=0.2$. We note that for $\Delta \omega \rightarrow 0$ the saddle-node bifurcation line always goes to vanishing noise intensity $D=0$, independently of $\Delta K$. Below the saddle-node curve, the excitable elements are resting and do not fire. For increasing $\Delta K$, the saddle-node line bends upwards, culminating in a Bogdanov-Takens bifurcation (BT), which is located at an intersection of the Hopf and the saddle-node lines. From the BT a homoclinic bifurcation line emanates, which ultimately merges with the saddle-node curve (then called a SNIC bifurcation line), see the insets in Figure 1. We calculate the homoclinic bifurcations as follows. Starting at the BT we continue the Hopf bifurcation for some time steps, then switch the continuation to the limit cycle while tracking the period with the noise intensity $D$ as the control parameter. At the homoclinic bifurcation the period of the limit cycle diverges. We accept the $D$ values if they do not change anymore in the order of $10^{-4}$ upon approaching the divergence. The whole procedure is repeated until the homoclinic bifurcation line reaches the saddle-node curve. Both for $\Delta K=2$ and $\Delta K=6$ we save hereby in total eight pairs of $(\Delta \omega, D)$ and connect them by a line (see Fig. 1 ).

Importantly, the area between the saddle-node bifurcations and the homoclinic bifurcation line corresponds to bistable (hysteretic) dynamics. In particular, two qualitatively different bistable dynamics are separated by the Hopf bifurcation line; below it, the resting and the partially synchronized state coexist, whereas above there is a coexistence between two steady states, the resting and the incoherent state (compare with Ref. [31]).
Besides performing a bifurcation analysis, another way of characterizing the collective dynamics lies in calculating suitable order parameters. One of them is the classical Kuramoto order parameter, equations (12), (13), which measures how similar the phase variables are to each other. However, it is not sufficient here to consider this order parameter, because in case of slowly varying phases, it would attain large values [29]. In the extreme case of resting elements, the Kuramoto order parameter would be even equal to unity, exactly as in the perfectly synchronized case. In order to distinguish between the resting and the synchronized state, one therefore needs to introduce an order parameter that decreases, if the elements collectively slow down. We consider here the well-known order parameter introduced by Shinomoto and Kuramoto [29]:

$$
\zeta_{\omega, K}(t)=\left|\rho_{1}(t \mid \omega, K)-\overline{\rho_{1}(t \mid \omega, K)}\right|,
$$

where $\rho_{1}(t \mid \omega, K)=r_{\omega, K}(t) \exp \left[i \Theta_{\omega, K}(t)\right]$ is the first coefficient of the Fourier series expansion of the one-oscillator probability density (17).

From now on, if we do not indicate an explicit timedependence, we refer to long-time averages. In Figure 2, we show the long-time averaged order parameters for certain sets of parameters, along with the bifurcation values as they can be extracted from Figure 1. The three main regions mentioned for the bifurcation diagram 1 can be discriminated here as follows. While the Kuramoto order parameters are close to unity, and the Kuramoto-Shinomoto order parameters are nearly vanishing, the whole system is at rest, and single units do 

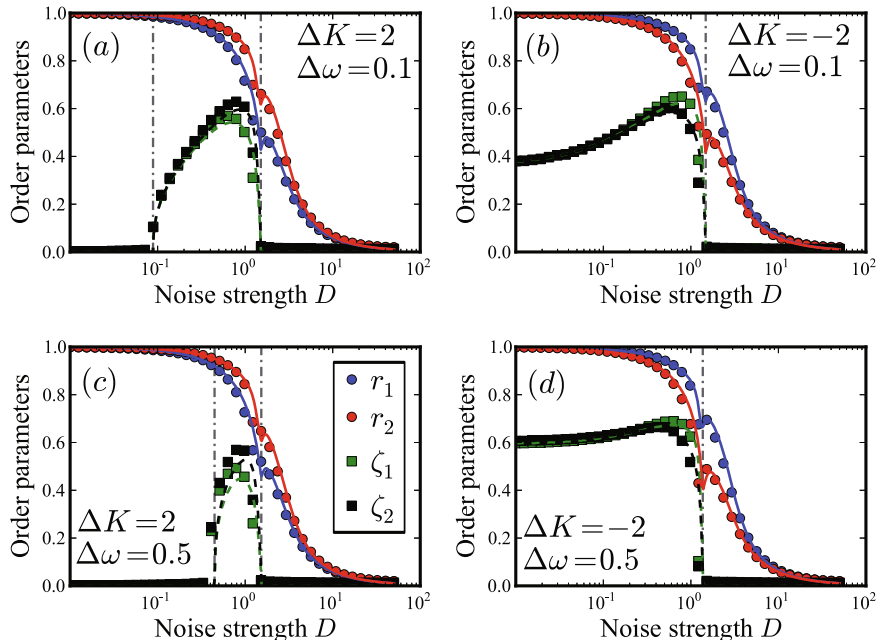

Fig. 2. Long-time averaged Kuramoto $\left(r_{1,2}\right)$ and KuramotoShinomoto order parameters $\left(\zeta_{1,2}\right.$, cf. Eq. (24)), theory (lines) vs. simulation (dots). Vertical dash-dotted lines correspond to Hopf and SNIC bifurcations, respectively, as it can be extracted from Figure 1. The average coupling strength is fixed at $K_{0}=4$.

not fire. A partially synchronized oscillation on the global scale is achieved if both the Kuramoto-Shinomoto and the Kuramoto order parameters attain non-zero values. The third region is characterized by vanishing KuramotoShinomoto and small Kuramoto order parameters. In this case, single units do fire, but in an incoherent way. In Figure 2, panels (a) and (c), the humps in the KuramotoShinomoto order parameters signal excitable behavior: for small noise intensities the population stays at rest, then at the SNIC bifurcation (first vertical dash-dotted line) one observes a transition to partial synchronization. Upon further increasing of the noise intensity the population becomes completely incoherent, which happens precisely at the Hopf bifurcation (second vertical dash-dotted line). Panels (b) and (d) show no excitable behavior, but only a single transition at the Hopf bifurcation from partial synchronization to incoherence. Noteworthy however, the Kuramoto-Shinomoto order parameters depend nonmonotonically on the noise intensity $D$, such that the highest level of synchronization is achieved at some non-zero noise intensity. The standard Kuramoto model cannot uncover this phenomenon.

Finally, one can observe that the theory agrees very well with the results from numerical simulations. Note that the log scale is not necessary to appreciate the accuracy, the latter is chosen in order to emphasize the humps in the excitable regime (compare with Ref. [63]). In Appendix $\mathrm{C}$ we discuss the accuracy in more detail. The numerical simulations are conducted by integrating the stochastic equations of motion (1) using the Heun scheme with time step 0.05 and considering populations of $N=10^{4}$ oscillators. Exactly one half of the population is assigned with frequencies and couplings $(\omega, K)=$ $\left(\omega_{1}, K_{1}\right)$, and the second half with $(\omega, K)=\left(\omega_{2}, K_{2}\right)$. Initial conditions of the phases $\phi_{i}(t=0)$ are Gaussian distributed with mean $m(t=0)=0$ and standard deviation
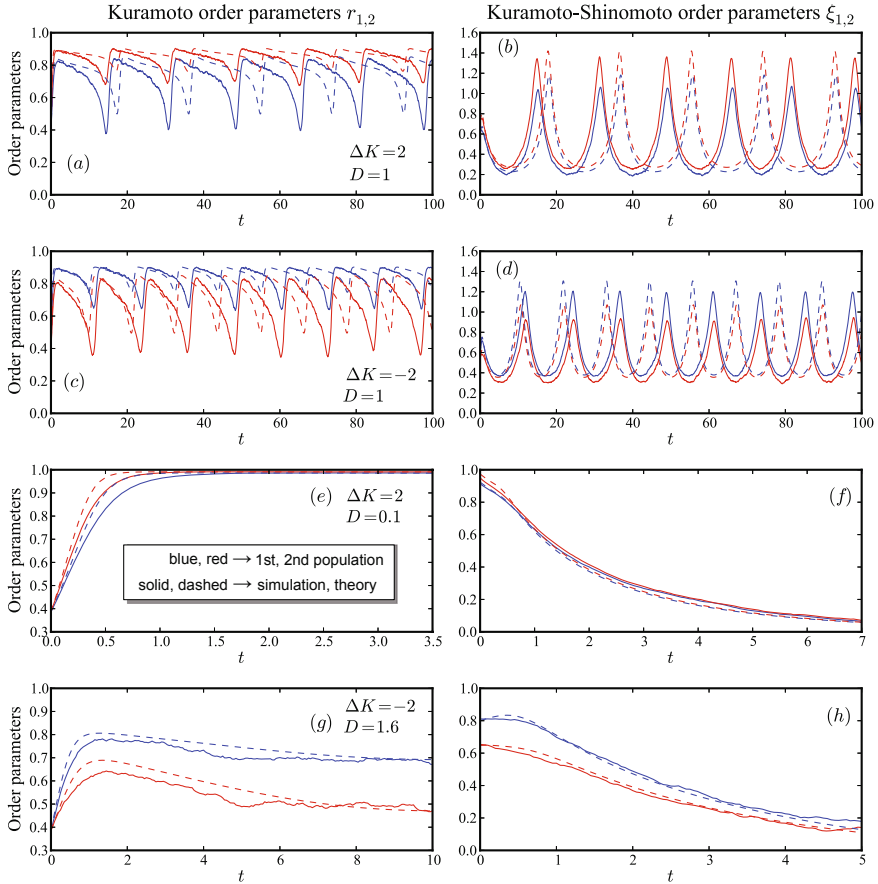

Fig. 3. Time-dependent order parameters, theory vs. simulation. The remaining parameters are frequency mismatch $\Delta \omega=0.5$ and average coupling strength $K_{0}=4$.

$\sigma(t=0)=\sqrt{2}$. Long-time averaged behavior of the order parameters is calculated by averaging the data between $t=[2500,5000]$. For the theoretical lines we integrated the reduced system (23) with the same integration parameters.

Figure 3 shows that even the time-dependent behavior is correctly described by the reduced system (23). The parameters can be compared with the bifurcation diagram (Fig. 1). Note that the Kuramoto-Shinomoto order parameters can exceed unity as a function of time (cf. Appendix B). Panels (a)-(d) reflect partially synchronized states, panels (e), (f) represent resting behavior, and panels (g), (h) correspond to incoherent dynamics. Apart from time shifts, the qualitative behavior is well predicted by the theory. In the collectively oscillating regime, the theoretical lines lag behind the simulation results for positive coupling mismatch $\Delta K$, but the order is reversed for negative $\Delta K$. Note that the initial values are not perfectly the same as a matter of fact. Finally, Figure 3 illustrates a fundamental feature of the active rotator model, namely the inhomogeneous evolution of the phases. Such a property results in periodically oscillating mean-field amplitudes and order parameters under partial synchronization, see panels (a)-(d). Moreover, in the incoherent regime the classical Kuramoto order parameter does not vanish, see panel (g).

\section{Conclusion}

In this paper we have studied the active rotator model [29] with distributed natural frequencies and 
coupling strengths. The crucial parameter in such excitable systems is the noise intensity $[41,42]$. In the infinite system-size limit, we have first derived the exact mean-field description. Assuming then that the phases in each set of oscillators with the same natural frequency and coupling strength obey a Gaussian distribution with timedependent cumulants, we have found a representation of the system that permits exemplary scenarios composed of a few differential equations. We have used this approach to analyze a mixed population, where one half has been chosen to be excitable, whereas the other half has been in a self-oscillatory state. The distinction depends on whether the natural frequency lies below or above the excitation threshold, respectively. Moreover, the elements of the two subpopulations have differed in their individual coupling strengths. In this way we have investigated how frequency and coupling mismatches affect the collective dynamics. In particular, we have performed a numerical bifurcation analysis in the plane spanned by the noise intensity and the frequency mismatch and have shown how these diagrams change as a function of the coupling mismatch. We have found that both large frequency and coupling mismatches impede the emergence of synchronized oscillations. This is consistent with the common finding that oscillatory units which are more distinct, are harder to synchronize. Most intriguingly however, we have found that excitability in the whole system is only present, if the excitable elements have a weaker coupling than the self-oscillatory ones. In other words, a positive couplingfrequency correlation is necessary to cause the excitable behavior in the mixed population. We have further found that bistability between various collective behaviors is only possible if the positive coupling-frequency correlation is strong enough. Such a phenomenon was previously reported only for systems without excitable dynamics (see e.g. Refs. $[11,21,24])$. The embedded self-oscillatory units considered here can be regarded as pacemaker cells in neuronal $[2]$ or cardiac $[6,8,9]$ tissues. Hence, we believe that the work presented here contributes to a better understanding of the collective dynamics observed in those systems. Finally, our work may provide a new perspective on the emergence of excitable behavior on the global scale, as it is observed e.g. in nonlinear optical cavities [64]. It would be interesting to further analyze effects of asymmetries in the natural frequencies and the coupling strengths, as it was done e.g. in reference [61] for a deterministic system. Moreover, one should also examine the situation where individual coupling strengths appear not outside but inside the coupling term, or where the interactions are allowed to be repulsive (see [65]).

B.S. thanks M.A. Zaks for helpful discussions. B.S. further acknowledges support from the Deutsche Forschungsgemeinschaft (GRK1589/1). L.SG. acknowledges the Bernstein Center for Computational Neuroscience Berlin (project A3). T.K.DM.P. acknowledges FAPESP (Grant 2012/22160-7) and IRTG 1740. F.A.R. acknowledges CNPq (Grant 305940/20104), Fapesp (Grant 2013/26416-9) and IRTG 1740 (DFG and
FAPESP) for financial support. J.K. acknowledges IRTG 1740 (DFG and FAPESP) for the sponsorship provided.

\section{Appendix A: The stochastic Kuramoto model with disordered coupling strengths}

Here we derive analytical results for the stochastic Kuramoto model (see Refs. [33,66-68] for the equivalent Brownian mean-field model) with distributed coupling strengths constituting the only source of quenched disorder, i.e.

$$
\dot{\phi}_{i}=\xi_{i}(t)+\frac{K_{i}}{N} \sum_{j=1}^{N} \sin \left(\phi_{j}-\phi_{i}\right),
$$

compare with equation (1). For an interesting recent study of the quenched limit $\xi_{i}(t) \rightarrow \omega_{i}$, we refer to [69].

Making use of equation (22), Section 4 , we get

$$
\left\{\begin{array}{l}
\dot{r}_{K}=-r_{K} D+\frac{1-r_{K}^{4}}{2} K\left\langle r_{K^{\prime}} \cos \left(\Theta_{K^{\prime}}-\Theta_{K}\right)\right\rangle, \\
\dot{\Theta}_{K}=\frac{r_{K}^{-1}+r_{K}^{3}}{2} K\left\langle r_{K^{\prime}} \sin \left(\Theta_{K^{\prime}}-\Theta_{K}\right)\right\rangle,
\end{array}\right.
$$

with a single average over the coupling strengths, $\langle\ldots\rangle \equiv$ $\int d K^{\prime} \ldots P\left(K^{\prime}\right)$. Applying the same arguments as in reference [33], one reveals that the critical noise intensity for the transition from partial synchronization to complete incoherence equals precisely $D_{c}=\left\langle K^{\prime}\right\rangle / 2$.

It is illustrative to come back to the mixed population case with two constituents, i.e.

$$
\left\{\begin{array}{l}
\dot{r}_{1}=-r_{1} D+\frac{1-r_{1}^{4}}{4} K_{1}\left[r_{1}+r_{2} \cos \left(\Theta_{2}-\Theta_{1}\right)\right] \\
\dot{\Theta}_{1}=\frac{r_{1}^{-1}+r_{1}^{3}}{4} K_{1} r_{2} \sin \left(\Theta_{2}-\Theta_{1}\right) ;
\end{array}\right.
$$

the equations for $\dot{r}_{2}$ and $\dot{\Theta}_{2}$ are obtained by replacing $1 \leftrightarrow 2$. It is possible to make progress in the stationary regime, $t \rightarrow \infty$, where the derivatives with respect to time vanish. In order that $\dot{\Theta}_{1}=0$, either $K_{1}$ or $r_{2}$ has to vanish or it must hold $\Theta_{2}=\Theta_{1}+m \pi, m \in \mathbb{Z}$. The first two choices need not to be considered, if we are interested in the partially synchronized state. Then from imposing $\dot{r}_{1}=0$ it directly follows that (note that the mean-field amplitude is a non-negative quantity)

$$
r_{2}=r_{1}\left(\frac{4 D}{\left(1-r_{1}^{4}\right) K_{1}}-1\right) \text {. }
$$

The analogous result follows for $r_{1}$ with the replacement $1 \leftrightarrow 2$. This is an interesting result per se, as it analytically relates the long-time levels of synchronization in the two subpopulations. Note that $r_{1,2}=1$ is achievable only for $D=0$ or $K_{1,2} \rightarrow \infty$, respectively. The two coupled equations for $r_{1,2}$ (A.4) can be solved simultaneously in a numerical way. The results are depicted in Figure A.1. The order parameter for the first subpopulation with the 


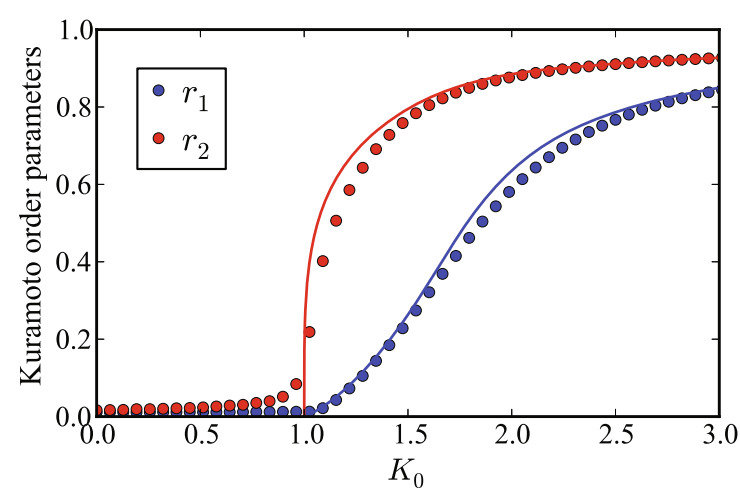

Fig. A.1. Stationary Kuramoto order parameters for the two subpopulations, theory (lines, cf. Eq. (A.4)) vs. simulation of full dynamics (dots, cf. Eq. (A.1)). Coupling mismatch equals $\Delta K=2$ and noise intensity $D=0.5$.

smaller coupling strength shows an anomalous scaling beyond the critical value, clearly different from the normal square-root scaling; apparently it does not even follow a critical power-law, but rather shows an exponential scaling. As a consequence, slightly above the critical coupling, one observes chimera-like states, where one subpopulation shows significant synchronization, while at the same time the other one stays almost incoherent. This scenario is reminiscent of what has been found in references [53,54]. We expect this to be a promising direction for future studies.

\section{Appendix B: A geometric view of the Kuramoto-Shinomoto order parameter}

Let us first repeat the definition of the KuramotoShinomoto order parameter (for simplicity, we neglect here the subdivision into distinct sets of natural frequencies and couplings):

$$
\zeta(t)=\left|\rho_{1}(t)-\overline{\rho_{1}(t)}\right|
$$

where $\rho_{1}(t)=r(t) \exp [i \Theta(t)]$. Now in the complex plane, $\zeta(t)$ corresponds to the length of one of the diagonals in the parallelogram that is spanned by the vectors $\rho_{1}(t)$ and $-\overline{\rho_{1}(t)}$. This is visualized in Figure B.1, where for simplicity the vectors are denoted by their absolute values. Clearly, $\zeta(t)$ can be larger than unity. In fact, the maximal value is 2 . However, this can be true only in an infinitely small time period, because $\overline{\rho_{1}(t)}$ is the long-time average of $\rho_{1}(t)$. To make this point more illustrative, imagine that half of the time the Kuramoto order parameter $\rho_{1}(t)$ is given by some unit vector, and the other half of the time by the zero vector. Then the Kuramoto-Shinomoto order parameter $\zeta(t)$ will equal $1 / 2$ at all times (see Eq. (B.1) and Fig. B.1). It is straightforward to see in this manner that the long-time averaged Kuramoto-Shinomoto order parameter always lies between zero and unity.

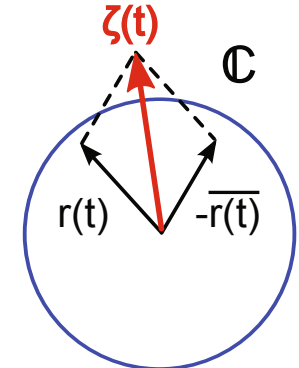

Fig. B.1. The unit circle in the complex plane helps to visualize the order parameters considered here (see Eq. (B.1)). Vectors are denoted by their absolute values.

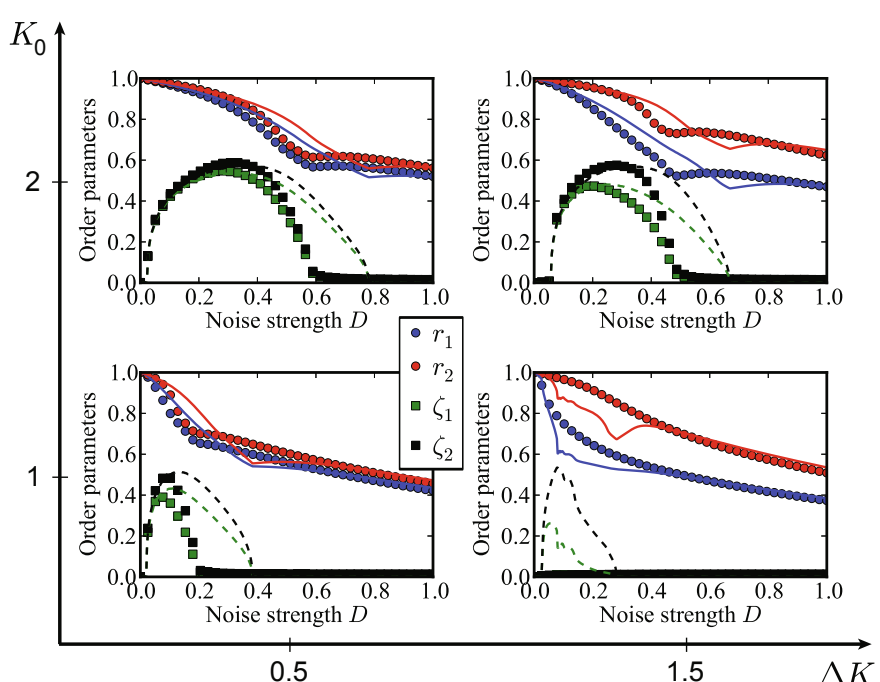

Fig. C.1. Long-time averaged order parameters as a function of the noise intensity are compared for different average coupling strengths $K_{0}$ and coupling mismatches $\Delta K$. All lines correspond to theoretical results cf. equation (23), while the symbols result from a simulation of the full dynamics (1). The frequency mismatch is fixed at $\Delta \omega=0.1$.

\section{Appendix C: On the accuracy of the Gaussian approximation (GA)}

It is well-known that a small noise intensity favors the GA, see e.g. [30]. In the recent paper [33] a systematic examination of the GA was carried out for the stochastic Kuramoto model. It was found that the critical coupling strength for the onset of synchronization is exactly recovered. Also below and sufficiently above (twice as much) the critical value, the GA is highly accurate. Here we proceed to show that a large coupling mismatch deteriorates the accuracy of the GA, in particular if the average coupling strength is small. In fact, the combination of large coupling mismatch $\Delta K$ and small average coupling strength $K_{0}$ is the only case where we find qualitative disagreement. Large noise intensities merely decrease the quantitative agreement and the frequency mismatch alone does not cause any inaccuracies. In Figure C.1 we depict the appearance of the qualitative disagreement for large $\Delta K$, but 
small $K_{0}$. There, the theory predicts excitable behavior, which is not reproduced by numerical simulations of the full system. The disagreement seems to be accompanied by an additional wiggle in the theoretical curves. Furthermore, one can clearly see the increased quantitative discrepancy for larger noise intensities $D$. Correspondingly, the saddle-node bifurcation is in general better reflected by the theory than the Hopf bifurcation in the excitable system. For very large noise intensities, i.e. in the incoherent state, the agreement between theory and simulations is recovered.

\section{References}

1. L. Glass, Nature 410, 277 (2001)

2. G. Buzsáki, Rhythms of the Brain (Oxford University Press, 2006)

3. D.E. Postnov, D.D. Postnov, L. Schimansky-Geier, Brain Research 1434, 200 (2012)

4. T.B. Luke, E. Barreto, P. So, Neural Comput. 25, 3207 (2013)

5. S. Luccioli, E. Ben-Jacob, A. Barzilai, P. Bonifazi, A. Torcini, PLoS Comput. Biol. (submitted) (2014)

6. D. DiFrancesco, Annu. Rev. Physiol. 55, 455 (1993)

7. T.K. Shajahan, B. Borek, A. Shrier, L. Glass, New J. Phys. 15, 023028 (2013)

8. A. Karma, Annu. Rev. Condens. Matter Phys. 4, 313 (2013)

9. Z. Qu, G. Hu, A. Garfinkel, J.N. Weiss, Phys. Rep. (2014), DOI : $10.1016 / \mathrm{j} \cdot$ physrep. 2014.05.002

10. M. Brede, Phys. Lett. A 372, 2618 (2008)

11. J. Gómez-Gardenes, S. Gómez, A. Arenas, Y. Moreno, Phys. Rev. Lett. 106, 128701 (2011)

12. T.K.D. Peron, F.A. Rodrigues, Phys. Rev. E 86, 056108 (2012)

13. I. Leyva, R. Sevilla-Escoboza, J.M. Buldú, I. SendiñaNadal, J. Gómez-Gardeñes, A. Arenas, Y. Moreno, S. Gómez, R. Jaimes-Reátegui, S. Boccaletti, Phys. Rev. Lett. 108, 168702 (2012)

14. B.C. Coutinho, A.V. Goltsev, S.N. Dorogovtsev, J.F.F. Mendes, Phys. Rev. E 87, 032106 (2013)

15. B. Sonnenschein, F. Sagués, L. Schimansky-Geier, Eur. Phys. J. B 86, 12 (2013)

16. P.S. Skardal, J. Sun, D. Taylor, J.G. Restrepo, Europhys. Lett. 101, 20001 (2013)

17. P. Li, K. Zhang, X. Xu, J. Zhang, M. Small, Phys. Rev. E 87, 042803 (2013)

18. G. Su, Z. Ruan, S. Guan, Z. Liu, Europhys. Lett. 103, $48004(2013)$

19. P. Ji, T.K.D. Peron, P.J. Menck, F.A. Rodrigues, J. Kurths, Phys. Rev. Lett. 110, 218701 (2013)

20. L. Zhu, L. Tian, D. Shi, Phys. Rev. E 88, 042921 (2013)

21. X. Zhang, X. Hu, J. Kurths, Z. Liu, Phys. Rev. E 88, 010802(R) (2013)

22. I. Leyva, A. Navas, I. Sendiña-Nadal, J.A. Almendral, J.M. Buldú, M. Zanin, D. Papo, S. Boccaletti, Sci. Rep. 3, 1281 (2013)

23. L. Zhu, L. Tian, D. Shi, Eur. Phys. J. B 86, 451 (2013)

24. H. Chen, G. He, F. Huang, C. Shen, Z. Hou, Chaos 23, 033124 (2013)
25. Y. Zou, T. Pereira, M. Small, Z. Liu, J. Kurths, Phys. Rev. Lett. 112, 114102 (2014)

26. P.S. Skardal, A. Arenas, Phys. Rev. E 89, 062811 (2014)

27. K. Mikkelsen, A. Imparato, A. Torcini, Phys. Rev. Lett. 110, 208101 (2013)

28. J.G. Orlandi, J. Soriano, E. Alvarez-Lacalle, S. Teller, J. Casademunt, Nat. Phys. 9, 582 (2013)

29. S. Shinomoto, Y. Kuramoto, Prog. Theor. Phys. 75, 1105 (1986)

30. C. Kurrer, K. Schulten, Phys. Rev. E 51, 6213 (1995)

31. M.A. Zaks, A.B. Neiman, S. Feistel, L. Schimansky-Geier, Phys. Rev. E 68, 066206 (2003)

32. B. Sonnenschein, M.A. Zaks, A.B. Neiman, L. Schimansky-Geier, Eur. Phys. J. Special Topics 222, 2517 (2013)

33. B. Sonnenschein, L. Schimansky-Geier, Phys. Rev. E 88, 052111 (2013)

34. S. Tanabe, K. Pakdaman, Phys. Rev. E 63, 031911 (2001)

35. M.A. Zaks, X. Sailer, L. Schimansky-Geier, A.B. Neiman, Chaos 15, 026117 (2005)

36. A.N. Burkitt, Biol. Cybern. 85, 247 (2001)

37. L.F. Lafuerza, R. Toral, J. Stat. Phys. 140, 917 (2010)

38. N. Burić, D. Ranković, K. Todorović, N. Vasović, Physica A 389, 3956 (2010)

39. I. Franović, K. Todorović, N. Vasović, N. Burić, Phys. Rev. E 87, $012922(2013)$

40. I. Franović, K. Todorović, N. Vasović, N. Burić, Phys. Rev. E 89, 022926 (2014)

41. V.S. Anishchenko, V. Astakhov, A. Neiman, T. Vadivasova, L. Schimansky-Geier, Nonlinear Dynamics of Chaotic and Stochastic Systems (Springer-Verlag, Berlin, 2007)

42. B. Lindner, J. García-Ojalvo, A. Neiman, L. SchimanskyGeier, Phys. Rep. 392, 321 (2004)

43. H. Risken, The Fokker-Planck Equation (Springer, 1996)

44. O. Faugeras, J. Touboul, B. Cessac, Front. Comput. Neurosci. 3, 1 (2009)

45. S. Mischler, C. Mouhot, Invent. Math. 193, 1 (2013)

46. M. Kac, in Proceedings of the Third Berkeley Symp. on Math. Statist. and Prob. (University of Calif. Press, 1956), Vol. 3, pp. 171-197

47. L.L. Bonilla, C.J.P. Vicente, J.M. Rubi, J. Stat. Phys. 70, $921(1993)$

48. E.J. Hildebrand, M.A. Buice, C.C. Chow, Phys. Rev. Lett. 98, 054101 (2007)

49. B. Sonnenschein, L. Schimansky-Geier, Phys. Rev. E 85, 051116 (2012)

50. T.D. Frank, Nonlinear Fokker-Planck Equations: Fundamentals and Applications (Springer, 2005)

51. E. Ott, T.M. Antonsen, Chaos 18, 037113 (2008)

52. E. Montbrió, J. Kurths, B. Blasius, Phys. Rev. E 70, $056125(2004)$

53. D.M. Abrams, R. Mirollo, S.H. Strogatz, D.A. Wiley, Phys. Rev. Lett. 101, 084103 (2008)

54. C.R. Laing, Chaos 19, 013113 (2009)

55. H. Hong, S.H. Strogatz, Phys. Rev. Lett. 106, 054102 (2011)

56. H. Hong, S.H. Strogatz, Phys. Rev. E 84, 046202 (2011)

57. D. Pazó, E. Montbrió, Phys. Rev. X 4, 011009 (2014)

58. H. Daido, K. Nakanishi, Phys. Rev. Lett. 93, 104101 (2004)

59. D. Pazó, E. Montbrió, Phys. Rev. E 73, 055202(R) (2006) 
60. H. Daido, A. Kasama, K. Nishio, Phys. Rev. E 88, 052907 (2013)

61. L.M. Alonso, G.B. Mindlin, Chaos 21, 023102 (2011)

62. A. Dhooge, W. Govaerts, Y.A. Kuznetsov, ACM Trans. Math. Softw. 29, 141 (2003)

63. C.J. Tessone, A. Scirè, R. Toral, P. Colet, Phys. Rev. E 75, 016203 (2007)

64. D. Gomila, M.A. Matías, P. Colet, Phys. Rev. Lett. 94, 063905 (2005)

65. H. Hong, S.H. Strogatz, Phys. Rev. E 85, 056210 (2012)

66. A. Pikovsky, S. Ruffo, Phys. Rev. E 59, 1633 (1999)
67. L. Bertini, G. Giacomin, K. Pakdaman, J. Stat. Phys. 138, $270(2010)$

68. P.H. Chavanis, Eur. Phys. J. B 87, 120 (2014)

69. D. Iatsenko, S. Petkoski, P.V.E. McClintock, A. Stefanovska, Phys. Rev. Lett. 110, 064101 (2013)

Open Access This is an open access article distributed under the terms of the Creative Commons Attribution License (http://creativecommons.org/licenses/by/4.0), which permits unrestricted use, distribution, and reproduction in any medium, provided the original work is properly cited. 